\title{
Gender Specific User Design Face vs. Interface
}

Ralph T. Reilly, University of Hartford, USA

\begin{abstract}
Human factors research has shown that the design and display of computer graphics plays a crucial role in the user operability of computer applications. In the future people will communicate with a face on the computer display screen. Already, advancements in artificial intelligence allow humans to "communicate" with computers through voice pattern recognition. Current work in artificial intelligence will allow the computer and user to "read" each other's facial expressions, understanding what can be communicated through facial mechanics. Research in facial emotion processing has suggested that gender plays a major role in the ability to correctly process human facial emotion.
\end{abstract}

Keywords: Facial mechanics, gender differences, distracter information, user design

\section{INTRODUCTION}

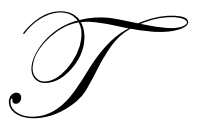

he face is an important avenue of nonverbal expression because of the amount of information it can convey, especially in a short period of time. Another important factor has been suggested by researchers who believe that there is a broader theoretical import to the relevance of facial behavior. Faces serve as signals for underlying emotional states through their portrayal of facial expression. The classification of facial expressions is a necessary prerequisite for the inference of emotion (Hansen 2003). Face processing engages several separately localized cognitive mechanisms. According to Hillger and Koenig (1991), there is evidence that separate left- and right-hemisphere mechanisms are involved in face processing. Some of them appear to be general-purpose visual mechanisms that are more efficient in the left-hemisphere, whereas others appear to be specialized (though not necessarily face-specific) mechanisms that are more efficient in the right hemisphere. The left-hemisphere seemed able to detect differences in single facial features (parsing). These mechanisms are probably engaged simultaneously when a stimulus is presented; the mechanism that is most appropriate for the judgment wins - i.e. produces a response faster and more accurately (Hillger and Koenig, 1991).

Gender Differences studies indicate that the female has a superior ability to recognize facial expressions of emotion. In examples conducted using photographs of male and female faces displaying anger, fear, sadness and disgust. These studies seem to further indicate that females are more accurate at revealing emotion than the male. (Zeng, Pantic, Roisman, Glenn, Huang, Thomas, 2009)

In Hall's (1984) review, she notes that females exceed males at all ages in their recognition of emotions and that women are also better senders (facial transmitters) of emotion than men (Buck, Miller, Caul, 1974; Buck, 1979; Hall, 1984; Kirouac and Dorè, 1985). Buck and Miller (1974) explored possible relationships between gender and personality variables, and the negative correlation between communication accuracy and physiological responding, as previously explained. The study demonstrated significant communication of emotion via facial expression using both the categorization and pleasantness measures of communication accuracy. The results indicated that the superior communication found among female pairs was due to the greater facial responsiveness of the female senders. Female senders demonstrated more accurate communication than male senders, but female observers were not found to be reliably more accurate than male observers. Females were also rated as being more facially expressive than males. Males had a tendency to be internalizers while females tended to be externalizers. This was related to cultural influences, since in our society males are generally discouraged more from overly expressing most emotions than are females. (Koch 2005) 
The idea that women respond to emotional stimuli by an activation of emotion processing systems, and men respond with a reactive inhibition is suggested in the research by Burton and Levy (1989). Their data showed that reaction times to negative emotions are faster overall than to positive emotions. Burton and Levy found that response times to negative emotions take longer in the right visual field than in the left visual field, whereas response times to positive emotions are longer in the left visual field. The effect was found to be significant among females but not among males. They suggested that the intensity of induced emotion may be greater for females than males due to the elaborative responses in females and the reactive, inhibitory responses in men.

In a face-to-face task, comparisons of a centrally presented and a laterally presented emotional face were required. The Emotional Valence (positive or negative response) by Visual Field Interaction (left visual field or right visual field) testing found that reaction times in the left visual field were faster for negative emotions presented in the left visual field. In addition, reaction times to positive emotions were faster when presented in the right visual field. An interaction of gender, task, and emotional valence was found. There were opposite effects for the two genders (Burton \& Levy). The reason for the opposite responses is that there is generally greater right hemisphere superiority for women for expressing facial emotion. The right cerebral hemisphere is specialized for interpretation and communication of emotion (Bourne, 2005). In the Harrison et al studies, evidence was found that indicated that left visual field presentations resulted in superior overall response times, irrespective of the affective valence of the stimulus of the gender of the subject. They also found evidence of overall better recognition for the positive emotions as compared to the negative emotions across visual half-fields. Additionally, men showed superior emotional identification with the left visual field while women did not. (Harrison et al, 1990).

Evidence was found that male subjects, as compared to female subjects, were selectively less sensitive to sad emotion in female faces. Female subjects were more sensitive overall to emotional expression in male faces than in female faces. In their study men and women differed in performance depending on the gender of the facial stimuli (Erwin, Gur, Gur, Skolnick, Mawhinney-Hee, \& Smailis, 1992).

\section{DISTRACTORS}

To achieve a communication function, facial movements have to be rapidly processed and decoded. (Kirouac \& Dorê, 1984) In their study Kirourac and Dorê used slides prepared in Ekman's Pictures of Facial Affect to test the responses of 20 university students. They used six emotions (happiness, surprise, disgust, anger, sadness and fear) and required each of the subjects to press one of six keys corresponding to the emotion displayed on the screen. Their results showed that humans could accurately abstract emotional information from facial stimuli available for extremely short intervals. Included in their procedures were visual masks (visual masking is a technique used in detection experiments that theoretically interferes with the processing of an image or the reduction of the visibility of one stimulus, called the target, by a spatio-temporally overlapping or adjacent second stimulus, called the mask) which quickly followed the target face (Fasel and Juergen, 2003).

\section{Discussion of Experiments}

The data was compiled and descriptive statistics were generated to test the dependent (subject's response time) versus independent (target affect, distractor affect, and gender of target) variables. Repeated-measures of analysis of variance suggested a statistical difference in judgment associated with the independent variables.

General results for female subjects, with variables, Distractor*Gender, yielded F(1,23) 4.704, p K .05, and with variables Target*Gender, yielded $\mathrm{F}(1,23)=4.043$, $\mathrm{p}<.06$. General results for male subjects, with variables, Target*Distractor*Gender, yielded $F(1,25) 0.369, \mathrm{p}<.06$, began to indicate a pattern wherein the distractor appeared to play a role in the test results. Both genders showed a quicker response time when processing a happy face of the same gender. Male response times were faster, overall, than female response times. Both male and female results showed the fastest times when the target face and the distractor face were both happy. It was not until analysis of the data was being done that the researcher discovered that no measures had been taken for the gender of the subject on an individualized basis. Thus, the reaction times recorded, while measured on an overall average for males and females as separate groups, had not been recorded for the gender of the subject. In addition, the researcher was not convinced that the population size of the study was large enough to adequately allow interpretation on a generalized basis. 
The experiment, therefore, was amended to include the gender of each subject as well as the gender of the target and distractor faces as well as to increase the population size. To increase the population size, an additional 50 subjects were chosen who had not taken part in the previous experiment. By disallowing repeat testing, the researcher wanted to avoid an unwanted familiarity with the same facial images. In addition, a third area of study was also included, the percentage of correct responses, allowing a more in-depth understanding of the subjects' recorded responses.

Results of the second experiment indicate that the distractor did, indeed, create a distraction for the subjects. General results for analysis of variance for percent correct with variables Subject Gender*Affect, yielded $\mathrm{F}(1,68)=7.800, \mathrm{p}<.01$. General results of analysis of variance for median reaction time with variables, Affect yielded, $\mathrm{F}(1,68) 13.10, \mathrm{p}<.001$ and with variables Target, yielded $\mathrm{F}(1,68)=8.457, \mathrm{p}<.005$ and with variables Affect*Tg, yielded $\mathrm{F}(1,68) 3.3805, \mathrm{p}<.01$. The initial design, therefore, should have been more likely to elicit an attentive search when responding to the target stimulus. Results further show that without the distractor, both male and female subjects processed happy male faces most quickly. Happy female faces were second in processing time. Without the distractor, the percentage correct for the affect of the target gender was higher for an angry male target than the other target affects.

ANOVA results for the affect of the target gender showed a significant difference when comparing male and female subject responses. While the male subject probability measured 0.4355 , the female subject probability measured only 0.0549 , not even close to the male subject results. What caused this much difference has not been determined by the research results. This is particularly interesting in light of the fact that this difference in variance was not evidenced as greatly in the first 100 subject results when the distractor was present.

In relating the results of this study to computer information systems, we will first examine cognitive psychology, or how we gain information, how much and how that information is transformed into knowledge and used to direct our attention and behavior. For the male subject, in all experiments, a happy male face was identified more quickly, regardless of distractor. More importantly, when the distractor was eliminated, the male response time dropped significantly. This would imply that a happy male computer image displayed to a male computer user would stimulate production and efficiency in tasks requiring artificial intelligence computer communications with a male subject user. Visual storage of information stemming from an ongoing "conversation" with a male human image on the screen would serve to keep the image on the screen longer, the added stimulus of auditory sensation would further enhance the storage of the information conveyed by the computer.

The perception of a familiar, comfortable face would allow the male user to become accustomed to "conversing" with a familiar face, forcing out the contextual cues and allowing concentration on the target, distinguished from competing normal variation.

From the results of Experiments Ia and Ib, the same would hold true for the female subject faced with a happy female computer displayed image in those instances where there is background information being transmitted (distractors). However, the results of Experiment II indicate that the male user will potentially respond more quickly than a female user, but that both will respond well to a happy male face displayed in a single display environment.

The human factors specialist should bear these findings in mind when engineering and designing not only the user interface to be used by the computer system, but the ergonomics of the system as well.

\section{CONCLUSIONS}

Judgment of the system is based on the affect it produces in the user, be that male or female. In designing human-computer interactive systems, no longer is a simple Graphical User Interface (GUI) sufficient. The designer should engineer a system that is designed ergonomically to use the graphical human face and expression most likely to cause an efficient, pleasant response in the user. Given the design specifications (single point of focus versus background distracter information) and the user community (male or female), the computer-displayed face would vary dependent solely on the purpose and user of the specific application. 


\section{REFERENCES}

1. Bourne VJ (2005) Lateralised processing of positive facial emotion: sex differences in strength of hemispheric dominance. Neuropsychologia 43: 953-956 Kelley, J.R., \& Spoor, J.R. (2005).

2. $\quad$ Bruce, V., (1988). Recognizing Faces. London: Lawrence Erlbaum Associates, Publishers.

3. Buck, R. A., (1979). Measuring Individual Differences in the Nonverbal Communication of Affect: The Slide-Viewing Paradigm. Human Communication Research. 6, 47-57.

4. Buck, R., Miller, R. E., Caul, W. F., (1974). Sex, Personality, and Physiological Variables in the Communication of Affect Via Facial Expression. Journal of Personality and Social Psychology. 30, 5 87596. Johns Hopkins University Press, in press.

5. Burton, L. A., \& Levy, J., (1989). Sex Differences in the Lateralized Processing of Facial Emotion. Brain and Cognition 11, 210-228.

6. Ekman, P., \& Friesen W. V., (1978a). Manual for the Facial Action Coding System. Palo Alto, California: Consulting Psychologists Press.

7. Eknian, P., \& Freisen, W. V., (1978b). Investigators Guide to the Facial Action Coding System, part I and II. Palo Alto, California: Consulting Psychologist Press.

8. Ekman, P., Friesen, W. V., \& Ellsworth, P., (1971). Emotion In The Human Face: Guidelines For Research and A Review of Findings. New York: Pergamon Press.

9. Ekman, P., \& O'Sullivan, M., (1988). The Role of Context in Interpreting Facial Expression: Comment on Russell and Fehr. Journal of Experimental Psychology: General. 117, 86-88.

10. Erwin, B.. J.; Gur, R. C.; Gur, B.. E.; Skolnick, B.; Mawbinney-Bee, M.; Smailis, J., (1992). Facial Emotion Discrimination: I. Task Construction and Behavioral Findings in Normal Subjects. Psychiatry Research. 42, 231-240.

11. Fasel, B., Luettin, J.Juergen, Automatic Facial Expression Analysis: A Survey, $P R(36)$, No. 1, January 2003, pp. 259-275.

12. Hall, J. A., (1984). Nonverbal Sex Differences: Communication Accuracy and Expressive Style. Baltimore, MD: Johns Hopkins University Press.

13. Hansen (2003)Affect as Medium, or the 'Digital-Facial-Image' Journal of Visual Culture.2003; 2: 205-228

14. Harrison, D. W., Gorelczenko, P. M. , \& Cook, J., (1990). Sex Differences in the Functional Asymmetry for Facial Affect Perception. Intern. J. Neurosciences. 52, 11-16.

15. Hillger, L. A., Koenig, 0., (1991). Separable Mechanisms in Face Processing: Evidence from Hemispheric Specialization. Journal of Cognitive Neuroscience. 3(1) 42-58.

16. Kirouac, G., \& Dorê, F. V., (1985). Accuracy of the Judgment of Facial Expression of Emotions as a Function of Sex and Level of Education. Journal of Nonverbal Behavior. 9(1) 3-27

17. Koch, S. C. (2005). Evaluative affect display toward male and female group leaders. A replication and extension. Small Group Research, 36 (6).

18. Stanners, B.. F., Byrd, D. M., \& Gabriel, B.., (1985). The Time It Takes To Identify Facial Expressions: Effects Of Age, Gender of Subject, Sex of Sender, and Type Of Expression. Journal of Nonverbal Behavior 9(4), Winter 201-2 11

19. Zajonc, B.. B., (1980). Feeling and thinking: Preferences Need No Inferences. American Psychologist. 35, 151-175.

20. Zeng, Z.H.Zhi-Hong, Pantic, M.Maja, Roisman, G.I.Glenn I., Huang, T.S.Thomas S., A Survey of Affect Recognition Methods: Audio, Visual, and Spontaneous Expressions, PAMI(31), No. 1, January 2009, pp. 39-58. 